\title{
Harmonic Forms on Manifolds with Non-Negative Bakry-Émery-Ricci Curvature
}

\author{
Matheus Vieira
}

\begin{abstract}
In this paper we prove that on a complete smooth metric measure space with non-negative Bakry-Émery-Ricci curvature if the space of weighted $L^{2}$ harmonic one-forms is non-trivial then the weighted volume of the manifold is finite and universal cover of the manifold splits isometrically as the product of the real line with an hypersurface.
\end{abstract}

Keywords: Harmonic forms, Non-negative Bakry-Émery-Ricci curvature, Smooth metric measure spaces.

\section{Introduction}

The theory of $L^{2}$ harmonic forms together with harmonic functions has been used to study the geometry and topology of complete manifolds (for example in $[8,3,9,10,5])$. The theory of smooth metric measure spaces has also been attracting some interest due to its connection with the Ricci flow (see [2]) and as an independent research topic: there are works about volume estimates [16, 6], essential spectrum of the drifted Laplacian [15], etc. In [11] Lott discussed (among other things) the topology of compact smooth metric measure spaces with non-negative Bakry-Émery-Ricci curvature using harmonic forms, and in [12] Munteanu and Wang obtained geometrical and topological results when these manifolds are non-compact using harmonic functions (see also [13, 14]). In this paper we investigate harmonic forms on non-compact smooth metric measure spaces with non-negative Bakry-Émery-Ricci curvature.

Recall that a smooth metric measure space $\left(M, g, e^{-f} d v\right)$ is a Riemannian manifold $(M, g)$ together with a smooth function $f$ and a measure $e^{-f} d v$. The Bakry-Émery-Ricci curvature is defined by the formula

$$
\operatorname{Ric}_{f}=\operatorname{Ric}+\operatorname{Hess} f .
$$

A differential form $\omega$ is called an $L_{f}^{2}$ differential form if

$$
\int_{M}|\omega|^{2} e^{-f} d v<\infty
$$


It is well known that the formal adjoint of the exterior derivative $d$ with respect to the $L_{f}^{2}$ inner product is given by the formula

$$
\delta_{f}=\delta+\iota_{\nabla f}
$$

where $\iota_{\nabla f}$ denotes the interior product with the vector field $\nabla f$ (see [1] or Lemma 2.1). The $f$-Hodge Laplacian is defined by the formula

$$
\Delta_{f}=-\left(d \delta_{f}+\delta_{f} d\right) .
$$

The space of $L_{f}^{2}$ harmonic one-forms is the set of all $L_{f}^{2}$ one-forms $\omega$ satisfying the equation

$$
\Delta_{f} \omega=0 .
$$

In this article we prove the following result (Theorem 4.1).

Theorem 1.1. Let $\left(M^{n}, g, e^{-f} d v\right)$ be a complete non-compact smooth metric measure space with non-negative Bakry-Émery-Ricci curvature. If the space of $L_{f}^{2}$ harmonic one-forms is non-trivial then the weighted volume of $M^{n}$ is finite, that is

$$
\operatorname{vol}_{f}\left(M^{n}\right)=\int_{M^{n}} e^{-f} d v<\infty,
$$

and the universal covering splits isometrically as $\tilde{M}^{n}=\mathbb{R} \times N^{n-1}$.

A corresponding result for compact manifolds is discussed in [11] (Theorem 1 , item 3$)$.

In particular we obtain the following result (Corollary 4.2) concerning the vanishing of $L_{f}^{2}$ harmonic forms when the Bakry-Émery-Ricci curvature is nonnegative and the first eigenvalue of the $f$-Laplacian is positive, which applies to non-trivial gradient Ricci steady solitons (see Corolary 4.3).

Corollary 1.2. Let $\left(M, g, e^{-f} d v\right)$ be a complete non-compact smooth metric measure space with non-negative Bakry-Émery-Ricci curvature. If the first eigenvalue of the $f$-Laplacian is positive then the space of $L_{f}^{2}$ harmonic oneforms is trivial.

We also obtain the following vanishing result (Corollary 4.4) for the space of $L_{f}^{2}$ harmonic forms when the function $f$ is bounded.

Corollary 1.3. Let $\left(M, g, e^{-f} d v\right)$ be a complete non-compact smooth metric measure space with non-negative Bakry-Émery-Ricci curvature. If the function $f$ is bounded then the space of $L_{f}^{2}$ harmonic one-forms is trivial.

In section 2 we prove that $L_{f}^{2}$ harmonic forms of any degree are closed and co-closed, in section 3 we prove Bochner's formula and Kato's inequality for $L_{f}^{2}$ harmonic one-forms, in section 4 we prove Theorem 4.1 and some corollaries.

The author wishes to thank his thesis advisor and the referee for many helpful suggestions. This work is part of the author's Ph.D. thesis, written under the supervision of Detang Zhou at Universidade Federal Fluminense. 


\section{$2 \quad L_{f}^{2}$ Harmonic Forms are Closed and Co-Closed}

In this section we extend to smooth metric measure spaces the known fact that

$L^{2}$ harmonic forms of any degree are closed and co-closed.

Recall that the dot product of differential forms (of the same degree) on a Riemannian manifold with a volume element $d v$ is defined by

$$
(\omega \cdot \eta) d v=\omega \wedge * \eta
$$

The $L_{f}^{2}$ inner product of $L_{f}^{2}$ differential forms is defined by

$$
(\omega, \eta)_{L_{f}^{2}(M)}=\int_{M} \omega \cdot \eta e^{-f} d v
$$

Notice that the $L_{f}^{2}$ inner product is also defined between any two differential forms if one of them is compactly supported.

First we prove for completeness the known fact that $\delta_{f}$ is the formal adjoint of $d$.

Lemma 2.1. Let $\omega$ be a $(p-1)$-form and $\eta$ be a p-form on a smooth metric measure space $\left(M, g, e^{-f} d v\right)$. If one of these differential forms is compactly supported then the following identity holds

$$
(d \omega, \eta)_{L_{f}^{2}(M)}=\left(\omega, \delta_{f} \eta\right)_{L_{f}^{2}(M)}
$$

Proof. Assume one of these differential forms compactly supported, say $\omega$. We have

$$
\begin{aligned}
(d \omega, \eta)_{L_{f}^{2}(M)} & =\int_{M} d \omega \cdot \eta e^{-f} d v \\
& =\int_{M} d \omega \wedge * \eta e^{-f} \\
& =\int_{M}\left(d\left(e^{-f} \omega\right) \wedge * \eta+e^{-f} d f \wedge \omega \wedge * \eta\right) \\
& =\int_{M} d\left(e^{-f} \omega \wedge * \eta\right)+\int_{M} \omega \wedge\left((-1)^{p} d * \eta+(-1)^{p-1} d f \wedge * \eta\right) e^{-f}
\end{aligned}
$$

Using Stokes theorem and the identities (see [7] for a list of formulas)

$$
(-1)^{p} d * \eta=* \delta \eta
$$

and

$$
d f \wedge * \eta=(-1)^{p-1} * \iota \nabla f \eta
$$

we get identity (2.1).

Now we prove that $L_{f}^{2}$ harmonic forms of any degree are closed and co-closed. Here we adapt from Carron [4]. 
Lemma 2.2. Every $L_{f}^{2}$ harmonic form (of any degree) on a complete smooth metric measure space $\left(M, g, e^{-f} d v\right)$ is closed and co-closed. In other words,

$$
d \omega=0
$$

and

$$
\delta_{f} \omega=0 .
$$

Proof. We can choose a smooth function $\phi$ on $M$ such that $\phi=1$ in $B_{R}, \phi=0$ in $M \backslash B_{2 R}$ and $|\nabla \phi| \leq \frac{2}{R}$ in $B_{2 R} \backslash B_{R}$. Here $B_{R}$ denotes a ball with center in a fixed point and radius $R$. We have

$$
\begin{aligned}
|d(\phi \omega)|_{L_{f}^{2}(M)}^{2} & =|d \phi \wedge \omega|_{L_{f}^{2}(M)}^{2}+\left(d \phi^{2} \wedge \omega, d \omega\right)_{L_{f}^{2}(M)}+|\phi d \omega|_{L_{f}^{2}(M)}^{2} \\
& =|d \phi \wedge \omega|_{L_{f}^{2}(M)}^{2}+\left(d\left(\phi^{2} \omega\right), d \omega\right)_{L_{f}^{2}(M)} \\
& =|d \phi \wedge \omega|_{L_{f}^{2}(M)}^{2}+\left(\phi^{2} \omega, \delta_{f} d \omega\right)_{L_{f}^{2}(M)} .
\end{aligned}
$$

Notice that we used Lemma 2.1 in equation (2.4). We also have the identity

$$
\begin{aligned}
\delta_{f}(\phi \omega) & =\delta(\phi \omega)+\iota_{\nabla f}(\phi \omega) \\
& =\phi \delta \omega-\iota_{\nabla \phi} \omega+\phi \iota_{\nabla f} \omega \\
& =\phi \delta_{f} \omega-\iota_{\nabla \phi} \omega,
\end{aligned}
$$

so

$$
\begin{aligned}
\left|\delta_{f}(\phi \omega)\right|_{L_{f}^{2}(M)}^{2} & =\left|\iota_{\nabla \phi} \omega\right|_{L_{f}^{2}(M)}^{2}-\left(\iota_{\nabla \phi^{2}} \omega, \delta_{f} \omega\right)_{L_{f}^{2}(M)}+\left|\phi \delta_{f} \omega\right|_{L_{f}^{2}(M)}^{2} \\
& =\left|\iota_{\nabla \phi} \omega\right|_{L_{f}^{2}(M)}^{2}+\left(\delta_{f}\left(\phi^{2} \omega\right), \delta_{f} \omega\right)_{L_{f}^{2}(M)} \\
& =\left|\iota_{\nabla \phi} \omega\right|_{L_{f}^{2}(M)}^{2}+\left(\phi^{2} \omega, d \delta_{f} \omega\right)_{L_{f}^{2}(M)} .
\end{aligned}
$$

Notice that we used Lemma 2.1 again in equation (2.6). Using the identity

$$
|d \phi \wedge \omega|^{2}+\left|\iota_{\nabla \phi} \omega\right|^{2}=|\nabla \phi|^{2}|\omega|^{2}
$$

and the assumption $\omega$ is an $L_{f}^{2}$ harmonic form, adding equations (2.4) and (2.6) we get

$$
|d(\phi \omega)|_{L_{f}^{2}(M)}^{2}+\left|\delta_{f}(\phi \omega)\right|_{L_{f}^{2}(M)}^{2}=\int_{M}|\nabla \phi|^{2}|\omega|^{2} e^{-f} d v
$$

Since $\omega$ is an $L_{f}^{2}$ differential form sending $R \rightarrow \infty$ in equation (2.7) we conclude that

$$
|d \omega|_{L_{f}^{2}(M)}^{2}+\left|\delta_{f} \omega\right|_{L_{f}^{2}(M)}^{2}=0,
$$

which implies identities (2.2) and (2.3). 


\section{Bochner's Formula and Kato's Inequality}

Let us recall classical Bochner's formula for one-forms

$$
\frac{1}{2} \Delta|\omega|^{2}=|\nabla \omega|^{2}+\Delta \omega \cdot \omega+\operatorname{Ric}(\omega, \omega)
$$

(here we use the same notation to represent the dual of a one-form) and Kato's inequality for $L^{2}$ harmonic one-forms

$$
|\nabla \omega|^{2} \geq\left.\frac{n}{n-1}|\nabla| \omega\right|^{2}
$$

In this section we extend these formulas to smooth metric measure spaces. First we extend Bochner's formula, which is also found in Lott's paper [11. (equation 2.10). We thank the referee for pointing out this reference.

Lemma 3.1. Let $\omega$ be a one-form on a smooth metric measure space $\left(M, g, e^{-f} d v\right)$. Then the following identity holds

$$
\frac{1}{2} \Delta_{f}|\omega|^{2}=|\nabla \omega|^{2}+\Delta_{f} \omega \cdot \omega+\operatorname{Ric}_{f}(\omega, \omega) .
$$

Proof. By a simple computation we have

$$
\Delta_{f}=\Delta-d \iota_{\nabla f}-\iota_{\nabla f} d
$$

so using the classical Bochner's formula for one-forms we get

$$
\begin{aligned}
\frac{1}{2} \Delta_{f}|\omega|^{2} & =\frac{1}{2} \Delta|\omega|^{2}-\frac{1}{2} \nabla f \cdot \nabla|\omega|^{2} \\
& =|\nabla \omega|^{2}+\Delta \omega \cdot \omega+\operatorname{Ric}(\omega, \omega)-\frac{1}{2} \nabla f \cdot \nabla|\omega|^{2} \\
& =|\nabla \omega|^{2}+\Delta_{f} \omega \cdot \omega+\operatorname{Ric}_{f}(\omega, \omega) \\
& -\frac{1}{2} \nabla f \cdot \nabla|\omega|^{2}-\operatorname{Hess} f(\omega, \omega)+d \iota_{\nabla f} \omega \cdot \omega+\iota_{\nabla f} d \omega \cdot \omega
\end{aligned}
$$

It remains to prove that

$$
-\frac{1}{2} \nabla f \cdot \nabla|\omega|^{2}-\operatorname{Hess} f(\omega, \omega)+d \iota_{\nabla f} \omega \cdot \omega+\iota_{\nabla f} d \omega \cdot \omega=0 .
$$

We can choose a local orthonormal basis $e_{1}, \ldots, e_{n}$ with dual basis $\theta_{1}, \ldots, \theta_{n}$ and assume the connection forms $\theta_{i j}$ vanish on a fixed point. Writing $\omega=\omega_{i} \theta_{i}$ we have

$$
\frac{1}{2} \nabla f \cdot \nabla|\omega|^{2}=f_{i} \omega_{j} \omega_{j i}
$$

and

$$
\operatorname{Hess} f(\omega, \omega)=\omega_{i} \omega_{j} f_{i j}
$$


Computing

$$
\begin{aligned}
d \iota_{\nabla f} \omega+\iota_{\nabla f} d \omega & =d\left(f_{i} \omega_{i}\right)+i_{\nabla f}\left(\omega_{i j} \theta_{j} \wedge \theta_{i}\right) \\
& =\omega_{i} d f_{i}+f_{i} d \omega_{i}+\omega_{i j} f_{j} \theta_{i}-\omega_{i j} f_{i} \theta_{j} \\
& =\omega_{i} f_{i j} \theta_{j}+f_{i} \omega_{i j} \theta_{j}+\omega_{i j} f_{j} \theta_{i}-\omega_{i j} f_{i} \theta_{j} \\
& =\left(\omega_{i} f_{i j}+f_{i} \omega_{j i}\right) \theta_{j},
\end{aligned}
$$

we obtain

$$
d \iota_{\nabla f} \omega \cdot \omega+\iota_{\nabla f} d \omega \cdot \omega=\omega_{i} \omega_{j} f_{i j}+f_{i} \omega_{j} \omega_{j i} .
$$

Equations (3.3), (3.4) and (3.5) imply equation (3.2).

Now we extend Kato's inequality.

Lemma 3.2. Let $\omega$ be an $L_{f}^{2}$ harmonic one-form on a smooth metric measure space $\left(M^{n}, g, e^{-f} d v\right)$. Then the following inequality holds

$$
|\nabla \omega|^{2} \geq \frac{1}{n-1}(|\nabla| \omega||-|\nabla f \cdot \omega|)^{2}+|\nabla| \omega||^{2} .
$$

Moreover, if equality holds in (3.6) then

$$
\nabla \omega=\left(\begin{array}{ccccc}
\lambda_{1} & 0 & \ldots & 0 & 0 \\
0 & \lambda_{2} & \ldots & 0 & 0 \\
\vdots & \vdots & \ddots & \vdots & \vdots \\
0 & 0 & \ldots & \lambda_{2} & 0 \\
0 & 0 & \ldots & 0 & \lambda_{2}
\end{array}\right)
$$

where $\lambda_{1}=\nabla f \cdot \omega-(n-1) \lambda_{2}$.

Proof. We can choose a local orthonormal basis $e_{1}, \ldots, e_{n}$ with dual basis $\theta_{1}, \ldots, \theta_{n}$. Writing

$$
\omega=\sum_{i=1}^{n} \omega_{i} \theta_{i}
$$

we have

$$
d \omega=\sum_{i, j=1}^{n} \omega_{i j} \theta_{j} \wedge \theta_{i}
$$

and

$$
\delta_{f} \omega=-\sum_{i=1}^{n} \omega_{i i}+\sum_{i=1}^{n} \omega_{i} f_{i} .
$$

By Lemma 2.2 we know that $L_{f}^{2}$ harmonic forms are closed and co-closed therefore

$$
\omega_{i j}=\omega_{j i}
$$

for $i, j=1, \ldots, n$ and

$$
\sum_{i=1}^{n} \omega_{i i}=\nabla f \cdot \omega
$$


In the rest of the proof we adapt from $\mathrm{Li}$ and Wang [9]. We can set $e_{1}=\frac{\omega}{|\omega|}$. Computing

$$
\begin{aligned}
\left.\left.|\nabla| \omega\right|^{2}\right|^{2} & =4 \sum_{i=1}^{n}\left(\omega_{1} \omega_{1 i}\right)^{2} \\
& =4|\omega|^{2} \sum_{i=1}^{n}\left(\omega_{1 i}\right)^{2},
\end{aligned}
$$

we obtain

$$
|\nabla| \omega||^{2}=\sum_{j=1}^{n} \omega_{1 j}^{2} .
$$

Using identities (3.8), (3.9) and (3.10) we get

$$
\begin{aligned}
|\nabla \omega|^{2} & =\omega_{11}^{2}+\sum_{j=2}^{n} \omega_{1 j}^{2}+\sum_{j=2}^{n} \omega_{j 1}^{2}+\sum_{i=2}^{n} \omega_{i i}^{2}+\sum_{i, j=2, i \neq j}^{n} \omega_{i j}^{2} \\
& \geq \omega_{11}^{2}+2 \sum_{j=2}^{n} \omega_{1 j}^{2}+\frac{1}{n-1}\left(\sum_{i=2}^{n} w_{i i}\right)^{2} \\
& =\omega_{11}^{2}+2 \sum_{j=2}^{n} \omega_{1 j}^{2}+\frac{1}{n-1}\left(-\omega_{11}+\nabla f \cdot \omega\right)^{2} \\
& =\frac{n}{n-1} \omega_{11}^{2}+2 \sum_{j=2}^{n} \omega_{1 j}^{2}+\frac{1}{n-1}(\nabla f \cdot \omega)^{2}-\frac{2}{n-1} \omega_{11} \nabla f \cdot \omega \\
& \geq\left.\frac{n}{n-1}|\nabla| \omega\right|^{2}+\frac{1}{n-1}(\nabla f \cdot \omega)^{2}-\frac{2}{n-1}|\nabla| \omega|| \cdot|\nabla f \cdot \omega| \\
& =\frac{1}{n-1}(|\nabla| \omega||-|\nabla f \cdot \omega|)^{2}+|\nabla| \omega||^{2} .
\end{aligned}
$$

Matrix (3.7) follows by analyzing intermediate inequalities.

Remark 3.3. The following inequality holds under the same assumptions of Lemma 3.2 .

$$
|\nabla \omega|^{2} \geq \frac{n}{n-1}|\nabla| \omega||^{2}+\frac{1}{n-1}(\nabla f \cdot \omega)^{2}-\frac{2}{n-1}|\omega|^{2}(\nabla f \cdot \omega) \nabla \omega(\omega, \omega) .
$$

\section{Non-Negative Bakry-Émery-Ricci Curvature}

In this section we prove some results concerning smooth metric measure spaces with non-negative Bakry-Émery-Ricci curvature.

First we prove the following theorem.

Theorem 4.1. Let $\left(M^{n}, g, e^{-f} d v\right)$ be a complete non-compact smooth metric measure space with non-negative Bakry-Émery-Ricci curvature. If the space of 
$L_{f}^{2}$ harmonic one-forms is non-trivial then the weighted volume of $M^{n}$ is finite, that is

$$
\operatorname{vol}_{f}\left(M^{n}\right)=\int_{M^{n}} e^{-f} d v<\infty,
$$

and the universal covering splits isometrically as $\tilde{M}^{n}=\mathbb{R} \times N^{n-1}$.

Proof. Fix a non-trivial $L_{f}^{2}$ harmonic one-form $\omega$ on $M$. Since the Bakry-ÉmeryRicci curvature is non-negative and the one-form $\omega$ is $f$-harmonic by Lemma 3.1 we have

$$
\frac{1}{2} \Delta_{f}|\omega|^{2} \geq|\nabla \omega|^{2}
$$

so

$$
|\omega| \Delta_{f}|\omega| \geq|\nabla \omega|^{2}-|\nabla| \omega||^{2}
$$

hence by lemma 3.2 we have

$$
|\omega| \Delta_{f}|\omega| \geq 0
$$

We can choose a smooth function $\phi$ on $M$ such that $\phi=1$ in $B_{R}, \phi=0$ in $M \backslash B_{2 R}$ and $|\nabla \phi| \leq \frac{2}{R}$ in $B_{2 R} \backslash B_{R}$. Here $B_{R}$ denotes a ball with center in a fixed point and radius $R$. Multiplying inequality (4.1) by $\phi^{2}$ and integrating by parts we get

$$
\int_{M}|\nabla| \omega||^{2} \phi^{2} e^{-f} d v \leq-2 \int_{M}|\omega| \phi \nabla|\omega| \cdot \nabla \phi e^{-f} d v .
$$

Using Cauchy-Schuwarz and Young's inequalities we get

$$
\frac{1}{2} \int_{M}|\nabla| \omega||^{2} \phi^{2} e^{-f} d v \leq 2 \int_{M}|\omega|^{2}|\nabla \phi|^{2} e^{-f} d v .
$$

Since $\omega$ is an $L_{f}^{2}$ differental one-form by the monotone convergence theorem sending $R \rightarrow \infty$ we get

$$
\frac{1}{2} \int_{M}|\nabla| \omega||^{2} e^{-f} d v \leq 0
$$

so the function $|\omega|$ is a constant $c$. Since

$$
\int_{M}|\omega|^{2} e^{-f} d v=c^{2} \operatorname{vol}_{f}(M)<\infty
$$

and $c \neq 0$ it follows that the weighted $\operatorname{volume~}_{\operatorname{vol}}(M)$ is finite. Now we claim that $\omega$ is parallel. Indeed, since $|\omega|$ is constant by Bochner's formula (Lemma 3.1) and the assumption in the Bakry-Émery-Ricci curvature we have

$$
0 \geq|\nabla \omega|^{2}
$$

so $\omega$ is parallel. Now the lifting of $\omega$ to the universal cover $\tilde{M}$ is a non-trivial parallel one-form, which concludes the proof by the de-Rham decomposition theorem. 
In particular we obtain the following result.

Corollary 4.2. Let $\left(M, g, e^{-f} d v\right)$ be a complete non-compact smooth metric measure space with non-negative Bakry-Émery-Ricci curvature. If the first eigenvalue of the $f$-Laplacian is positive then the space of $L_{f}^{2}$ harmonic oneforms is trivial.

Proof. By assumption the first eigenvalue of the $f$-Laplacian

$$
\lambda_{1}\left(\Delta_{f}\right)=\inf _{\phi \in C_{c}^{\infty}(M)} \frac{\int_{M}|\nabla \phi|^{2} e^{-f} d v}{\int_{M} \phi^{2} e^{-f} d v}
$$

is positive. We can choose a smooth function $\phi$ on $M$ such that $\phi=1$ in $B_{R}$, $\phi=0$ in $M \backslash B_{2 R}$ and $|\nabla \phi| \leq \frac{2}{R}$ in $B_{2 R} \backslash B_{R}$. Then

$$
\lambda_{1}\left(\Delta_{f}\right) \int_{M} \phi^{2} e^{-f} d v \leq \frac{4}{R^{2}} \operatorname{vol}_{f}(M) .
$$

We claim that $\operatorname{vol}_{f}(M)$ is not finite. Otherwise by the monotone convergence sending $R \rightarrow \infty$ we get $\lambda_{1}\left(\Delta_{f}\right)=0$, a contradiction. Now since $\operatorname{vol}_{f}(M)=\infty$ it follows from Theorem 4.1 that the space of $L_{f}^{2}$ harmonic forms is trivial.

Recall that a gradient steady Ricci soliton is a manifold $(M, g)$ together with a smooth function $f$ satisfying

$$
\operatorname{Ric}+\operatorname{Hess} f=0 .
$$

In this case, it is possible to prove that the scalar curvature $R$ is non-negative and there is a constant $a$ such that

$$
R+|\nabla f|^{2}=a
$$

(see [2]). It follows that $|\nabla f| \leq \sqrt{a}$, so the only non-trivial gradient steady Ricci solitons are those with $a>0$, which must be non-compact (see [2]). It was proved by Munteanu and Wang in 12 that the first eigenvalue of the $f$-Laplacian on non-trivial gradient steady Ricci solitions is positive, more precisely $\lambda_{1}\left(\Delta_{f}\right)=a^{2} / 4$, so Corollary 4.2 implies the following result.

Corollary 4.3. On a complete non-compact non-trivial gradient steady Ricci soliton $(M, g, f)$ the space of $L_{f}^{2}$ harmonic one-forms is trivial.

It was proved by Wei-Wyllie in [16] that on a complete non-compact smooth metric measure space $\left(M, g, e^{-f} d v\right)$ if the function $f$ is bounded and the BakryÉmery-Ricci curvature is non-negative then the weighted volume $\operatorname{vol}_{f}(M)$ is infinite, so Theorem 4.1 has the following consequence.

Corollary 4.4. Let $\left(M, g, e^{-f} d v\right)$ be a complete non-compact smooth metric measure space with non-negative Bakry-Émery-Ricci curvature. If the function $f$ is bounded then the space of $L_{f}^{2}$ harmonic one-forms is trivial. 


\section{References}

[1] E. Bueler. The heat kernel weighted Hodge Laplacian on noncompact manifolds. Trans. Amer. Math. Soc. 351 (1999), no. 2, 683-713.

[2] H-D. Cao. Recent progress on Ricci solitons. Adv. Lect. Math. (ALM), 11, Int. Press, Somerville, MA, 2010.

[3] H-D. Cao, Y. Shen and S. H. Zhu. The structure of stable minimal hypersurfaces in $R^{n+1}$. Math. Res. Lett. 4 (1997), no. 5, 637-644.

[4] G. Carron. $L^{2}$ harmonic forms on non compact manifolds. arXiv:0704.3194 (2007).

[5] X. Cheng, L. Cheung and D. Zhou. The structure of weakly stable constant mean curvature hypersurfaces. Tohoku Math. J. (2) 60 (2008), no. 1, 101-121.

[6] X. Cheng and D. Zhou. Volume estimate about shrinkers. Proc. Amer. Math. Soc. 141 (2013), no. 2, 687-696.

[7] S. Kong, P. Li and D. Zhou. Spectrum of the Laplacian on quaternionic Kähler manifolds. J. Differential Geom. 78 (2008), no. 2, 295-332.

[8] P. Li and L-F. Tam. Harmonic functions and the structure of complete manifolds. J. Differential Geom. 35 (1992), no. 2, 359-383.

[9] P. Li and J. Wang. Complete manifolds with positive spectrum. J. Differential Geom. 58 (2001), no. 3, 501-534.

[10] P. Li and J. Wang. Complete manifolds with positive spectrum, II. J. Differential Geom. 62 (2002), no. 1, 143-162.

[11] J. Lott. Some geometric properties of the Bakry-Émery-Ricci tensor. Comment. Math. Helv. 78 (2003), no. 4, 865-883.

[12] O. Munteanu and J. Wang. Smooth metric measure spaces with nonnegative curvature. Comm. Anal. Geom. 19 (2011), no. 3, 451-486.

[13] O. Munteanu and J. Wang. Analysis of weighted Laplacian and applications to Ricci solitons. Comm. Anal. Geom. 20 (2012), no. 1, 55-94.

[14] O. Munteanu and J. Wang. Geometry of manifolds with densities. arXiv:1211.3996 (2012).

[15] L. Silvares. On the essential spectrum of the Laplacian and the drifted Laplacian. arXiv:1302.1834 (2013).

[16] G. Wei and W. Wylie. Comparison geometry for the Bakry-Émery Ricci tensor. J. Differential Geom. 83 (2009), no. 2, 377-405. 
Departamento de Matemática, Universidade Federal do Espírito Santo, 29075-910, Vitória, Brazil

matheus.vieira@ufes.br 